\title{
Oxidative Thymine Mutation in DNA: Water-Wire-Mediated Proton- Coupled Electron Transfer
}

\author{
Robert N. Barnett, ${ }^{\dagger}$ Joshy Joseph, ${ }^{\ddagger}, \#$ Uzi Landman, ${ }^{* \dagger}$ and Gary B. Schuster*, ${ }^{\dagger}$ \\ ${ }^{\dagger}$ School of Physics, Georgia Institute of Technology, Atlanta, Georgia 30332-0430, United States \\ ${ }^{\ddagger}$ School of Chemistry \& Biochemistry, Georgia Institute of Technology, Atlanta, Georgia 30332-0400, United States
}

Supporting Information

ABSTRACT: One-electron oxidation of A/T-rich DNA leads to mutations at thymine. Experimental investigation of DNA containing methyl-deuterated thymine reveals a large isotope effect establishing that cleavage of this carbon-hydrogen bond is involved in the rate-determining step of the reaction. Firstprinciples quantum calculations reveal that the radical cation (electron hole) generated by DNA oxidation, initially located on adenines, localizes on thymine as the proton is lost from the methyl group, demonstrating the role of proton-coupled

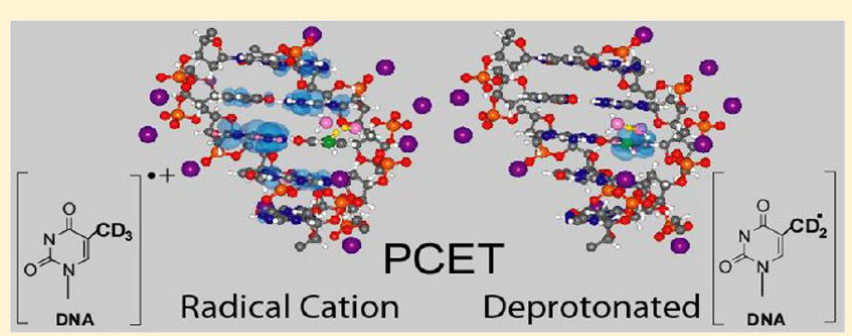
electron transfer (PCET) in thymine oxidation. Proton transport by structural diffusion along a segmented "water-wire" culminates in proton solvation in the hydration environment, serving as an entropic reservoir that inhibits reversal of the PCET process. These findings provide insight into mutations in A/T-rich DNA such as replication fork stalling that is implicated in early stage carcinogenesis.

\section{INTRODUCTION}

Proton-coupled electron transfer $(\text { PCET })^{1}$ is an essential component of several important biochemical processes including photosynthetic energy transduction, the formation of deoxyribonucleotides, ${ }^{2,3}$ and one-electron oxidation of DNA resulting in reactions of radical cations that damage nucleobases and generate mutations and malignancy. ${ }^{4,5}$ A defining characteristic of DNA oxidation has been the preferential generation of mutagenic lesions at guanines. ${ }^{6}$ However, we recently found that oxidation of duplex DNA comprised solely of $\mathrm{A} / \mathrm{T}$ base pairs results in ca. $95 \%$ of the observed reaction at thymine, and only $5 \%$ occurs at adenine. ${ }^{7}$ Significantly, replication fork stalling at fragile DNA sites that are rich in $\mathrm{A} / \mathrm{T}$ base pairs is implicated in chromosomal instability and early stage carcinogenesis. ${ }^{8,9}$ These fragile sites are regions where thymine mutations are the expected result of oneelectron oxidation of DNA. Further, it is known that thymine glycol, a product of thymine oxidation, causes replication stalling. ${ }^{10,11}$

The quantum mechanical PCET mechanism, with the dual involvement of two elementary particles (a proton and an electron), opens facile pathways for the oxidative reaction at thymine. Measurements reported here of a characteristically large kinetic isotope effect in the oxidation of DNA containing methyl-deuterated thymine confirm the rate-determining deprotonation step of this process. Quantum simulations reveal localization of the ionization hole on $\mathrm{T}$ simultaneously with proton loss that is facilitated by a hydrogen-bonded network of the DNA-water environment and by the long-range motion of a proton through a segmented molecular water "wire".
One-electron oxidation of duplex DNA introduces a radical cation (electron ionization hole) that may migrate hundreds of angstroms by a reversible hopping process before being trapped irreversibly by reaction. ${ }^{12,13}$ In typical DNA, containing all four common bases, reactions occur preferentially at guanine because holes pause briefly at thermodynamically favorable sites and guanine is the nucleobase with the lowest $E_{\text {ox }}{ }^{14}$ However, for duplexes containing only A/T base pairs, oxidative reaction occurs at thymine ${ }^{15}$ despite the fact that the population of the thymine radical cation in an $\mathrm{A} / \mathrm{T}$ base pair will be less than $1 \%$ of that at the adenine $\left(\Delta E_{\mathrm{ox}}=0.15 \mathrm{~V}\right.$ in water). The thymine oxidation products are formed by reaction of its 5-methyl group giving 5-hydroxymethylthymine and 5-formyluracil (see Figure 1), and by addition to its 5,6double bond to give cis- and trans-thymine glycols, the latter process is initiated by methyl group deprotonation at TT steps. ${ }^{7}$ The minor product from oxidation of A/T DNA is 8oxoadenine and possibly others that may be formed by deprotonation of the amino group of the adenine radical cation. ${ }^{16}$ The seemingly contradictory observation that reaction occurs primarily on a base with a higher $E_{\text {ox }}$ is reconciled by noting that product ratios depend on differences in the free energy of the transition states. ${ }^{17}$ Thus, the dominant reaction of radical cations at thymine in DNA signals the operation of a favorable pathway for this process. The discovery of that process is the focus of this investigation.

Characteristically large kinetic isotope effects are observed in reactions in which carbon-hydrogen bond cleavage occurs in

Received: November 16, 2012

Published: February 19, 2013 


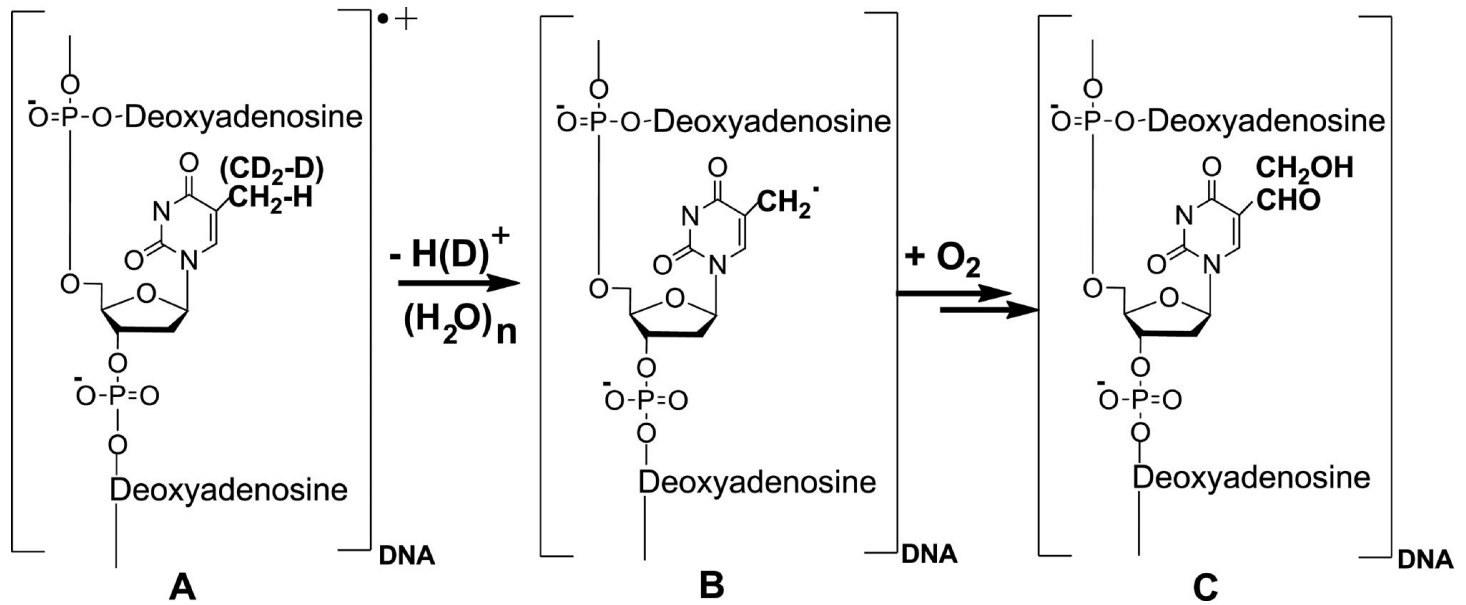

Figure 1. Radical cation reaction at thymine. (A) A radical cation (ionization hole) formed by the one-electron oxidation (ionization) of duplex DNA that is comprised exclusively of A/T base pairs. The figure shows a single thymine specifically and the case where the $\mathrm{CH}_{3}$ group of this thymine has been replaced by a $\mathrm{CD}_{3}$ group. (B) Loss of a proton (deuteron) from the 5-methyl group of the oxidized DNA generates a 5-thymidyl (or dideuteriothymidyl) radical. (C) The 5-thymidyl radical is trapped by reaction with molecular oxygen to generate, eventually, 5-formyluridine and 5-hydroxymethyluridine, the other observed products.

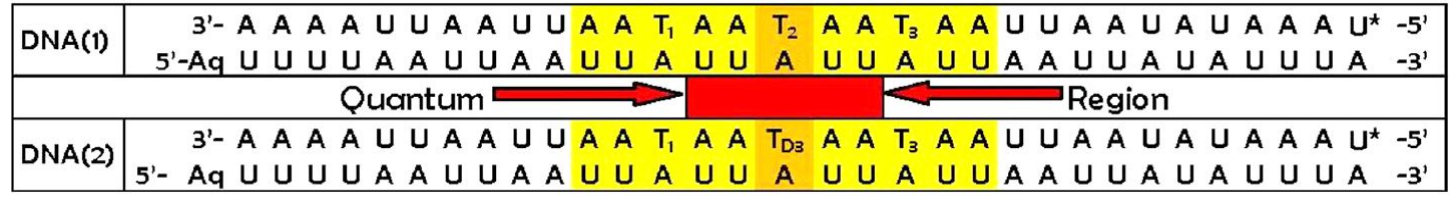

Figure 2. Structures of DNA sequences used in this study. Aq represents an anthraquinone photosensitizer that is covalently attached to the indicated $5^{\prime}$-terminus of the DNA oligomers through a dimethylene chain. ${ }^{43} \mathrm{~A}, \mathrm{U}, \mathrm{T}$, and $\mathrm{T}_{\mathrm{D} 3}$ represent 2-deoxyadenosine, 2-deoxyuridine, 2deoxythymine, and trideuteriothymidine, respectively. The position of ${ }^{32} \mathrm{P}$ label is shown by the asterisk $(*)$ on the $5^{\prime}-\mathrm{U}$. In the theoretical simulations (see below), the 11-base-pair yellow-colored central segment is considered. The region treated quantum mechanically is indicated by the red arrows, and the remaining six base pairs (three on each side of the quantum region) are treated classically.

the rate-determining step. ${ }^{18-20}$ In this work, we observe a large primary isotope effect from the one-electron oxidation of DNA containing a (trideuteriomethyl)thymidine $\left(\mathrm{T}_{\mathrm{D} 3}\right)$ in place of thymine. First-principles quantum mechanical simulations reveal that transfer of a proton from the methyl group of thymine to water in oxidized DNA localizes the hole (radical cation), initially situated primarily on adenines, onto the reacting thymine, thus constituting a PCET reaction.

This Article is organized as follows. The next section contains the results and the discussion of our investigations. First, we describe the DNA sequences that were examined, followed by an analysis of strand cleavage at the thymine nucleobases following loss of an electron from the DNA. The results reveal a large deuterium kinetic isotope effect showing that the deprotonation of the methyl group of the thymine radical cation to form the methyl radical is the rate-determining step in the conversion of thymine in DNA to its oxidation products. Next, we display and analyze the results of firstprinciples quantum mechanical simulations aimed at characterizing the effects of ionization on the electronic and atomic structures of the DNA molecule and its hydration environment. Creation of the radical cation (ionization hole) on the DNA by the removal of an electron is found to orientationally reorganize the water molecules near the DNA, localizing the hole predominantly on adenine bases neighboring the deprotonation (thymine) site. Computational exploration of the pathways for the thymine deprotonation process reveals a concerted bidirectional PCET mechanism involving passage over an energy barrier $\left(\Delta_{\mathrm{a}} \approx 0.85 \mathrm{eV}\right)$ that is strongly coupled with (simultaneous) transfer of the hole to the deprotonating $\mathrm{T}$ (a process that can be equivalently expressed as a concerted transfer of an electron from $\mathrm{T}$ to $\mathrm{A})$. This reaction step yields a Zundel ion $\left(\mathrm{H}_{5} \mathrm{O}_{2}^{+}\right)$, and subsequent barrierless proton transport from the reaction site into the bulk of the liquid environment proceeds via a segmented-water-wire mechanism (involving alternating $\mathrm{H}_{5} \mathrm{O}_{2}{ }^{+}$and $\mathrm{H}_{7} \mathrm{O}_{3}{ }^{+}$charged segments).

Following a brief Conclusion section where our findings are summarized, a description of the principal methods used is presented. This includes a discussion of pertinent details about the reorientational response of the hydration medium to the ionization of the DNA, and the dependence of the deprotonation PCET process on the degree of hole-localization in the initial ionized DNA configuration. A detailed account of the simulation methodology and system preparation procedures is presented in the Supporting Information.

\section{RESULTS AND DISCUSSION}

Experiment. The conversion of a thymine in DNA to its observed oxidation products is a complex, multistep process. Previous experiments on thymine oxidation in DNA have confirmed the central role for its 5-methyl group. ${ }^{7}$ We carried out a series of experiments designed to investigate the nature of the crucial steps in this process. DNA(1), shown in Figure 2, contains a central segment with the sequence AATAATAATAA that has three thymines separated by AA steps. In DNA(2) the middle $\mathrm{T}$ of this segment is replaced by $\mathrm{T}_{\mathrm{D} 3}$. We have shown previously that oxidative reaction is suppressed in DNA duplexes when a 2-deoxyuridine $(U)$ replaces a $T$ because $U$ 
lacks the methyl group required for reaction. ${ }^{7}$ In both $\mathrm{DNA}(1)$ and (2), all of the thymines other than the three in the central segments are replaced by uracils to increase the sensitivity of PAGE analysis of the effect of replacement of the central $T$ with $\mathrm{T}_{\mathrm{D} 3}$.

DNA(1) contains a covalently linked anthraquinone group (Aq) whose irradiation at $350 \mathrm{~nm}$ results in the one-electron oxidation of the DNA and injects a radical cation into the duplex. $^{21}$ Nucleobase radical cations are much stronger acids than their neutral counterparts. ${ }^{22}$ The irradiated samples of DNA(1) were treated with piperidine or endo III enzyme, which causes strand cleavage at the sites of mutated bases, ${ }^{23}$ and then were analyzed by gel electrophoresis. The amount of strand cleavage was quantified by phosphorimagery. The results are shown in Figure 3; they are essentially identical in the base and enzyme-treated experiments.

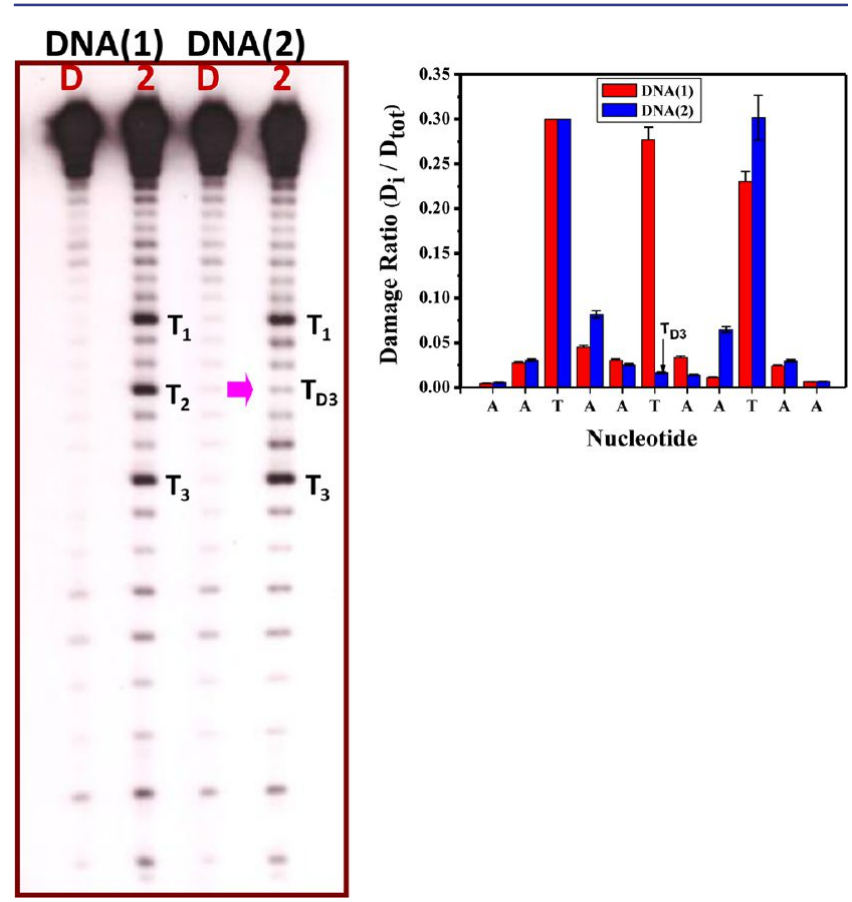

Figure 3. Autoradiograms of the PAGE gel following irradiation. The irradiated samples were treated with piperidine. DNA(1) and DNA(2) lanes labeled " $\mathrm{D}$ " are dark controls (no UV irradiation), and lanes labeled " 2 " are after 2 min of irradiation. The position of the $\mathrm{T}_{\mathrm{D} 3}$ is indicated by the purple arrow. The histogram on the right shows the amount of cleavage for the specific bases in the middle segment [AATAAT $\left.\left(\mathrm{T}_{\mathrm{D} 3}\right) \mathrm{AATAA}\right]$ of the DNA as compared to the total amount of strand cleavage. See Supporting Information E1.

The result of the one-electron oxidation of $\operatorname{DNA}(1)$ is extensive strand cleavage observed at the three thymine nucleobases in the central segment and a minor amount of cleavage at adenines. The relative amount of cleavage is independent of irradiation time at low conversion where there is a high probability of "single hits" on each DNA strand analyzed. The distance dependence for strand cleavage at the three thymines of $\mathrm{DNA}(1)$ is consistent with that observed in more extensive studies of this reaction. ${ }^{7}$ Clearly, as has been previously shown, UV irradiation of the Aq leads to injection of a radical cation (hole) into the DNA that migrates through the duplex until it is trapped in an irreversible reaction. ${ }^{7,12}$ In this case, reaction occurs primarily at thymine and yields products that give strand cleavage when treated with piperidine or endo III.

Similarly, analysis of the irradiation of DNA(2) reveals strand cleavage at $\mathrm{T} 1$ and $\mathrm{T} 3$ of the central segment, but there is no significant cleavage at the central position in which $T$ is replaced by $T_{D 3}$. The data are shown in Figure 3 both as a PAGE gel and as a histogram. ${ }^{24}$ Quantitative analysis of multiple independent samples reveals that the amount of strand cleavage at the $\mathrm{T}_{\mathrm{D} 3}$ of $\mathrm{DNA}(2)$ is statistically indistinguishable from background cleavage, which yields an isotope effect greater than $15 \pm 2$. This isotope effect shows that the deprotonation of the thymine radical cation is the ratedetermining step in the conversion of thymine in DNA to its oxidation products. ${ }^{20}$ The products formed in this process arise from trapping of the resulting thymine methyl radical by molecular oxygen and subsequent reactions of the intermediate hydroperoxy radical ${ }^{7}$ as shown schematically in Figure 1.

Theory. Fundamental insight into the mechanism underlying the oxidative thymine mutation (step A $\rightarrow$ B in Figure 1) was gained by extensive quantum mechanical first-principles molecular dynamics (FPMD, see Methods and Supporting Information S1) simulations of an 11-base-pair DNA duplex including sodium counterions and a set of hydrating water molecules, coinciding with the central segment of the DNA oligomers described above (see Figures 2 and 4). First, we equilibrated at room temperature the hydrated DNA oligomer (first as a neutral and subsequently as a singly charged molecule, see Methods and Supporting Information S1) using a prolonged constant-temperature (canonical) classical molecular dynamics (MD) simulation. Representative configurations selected from the equilibrium ensemble of the charged system were structurally optimized using FPMD simulations, with the five base-pair (double-strand) region (see Figures 2 and 4) as well as adjacent hydrating water molecules treated quantum mechanically while interacting with the other bases of the 11base-pair DNA oligomer (and the rest of the water environment was treated classically, see Methods and Supporting Information S1A). The resulting ground-state radical cation configurations served as samples for subsequent exploration of the deprotonation mechanism. Figure 4 shows one of these optimized configurations with all $\mathrm{Na}^{+}$counterions located near the backbone phosphate groups; the highlighted region comprises the (right) DNA strand containing the thymine ( $\mathrm{T}$, its complementary adenine $(\mathrm{A})$ is located on the other strand) whose methyl group (green sphere) is the deprotonation site. Also shown are water molecules (whose oxygen atoms are depicted in pink) and the phosphate group (the phosphorus atom colored orange and one of the oxygen atoms depicted in light blue) that participate in the proton-transfer processes; stereographic images of Figure 4 are shown in section S2 of the Supporting Information. This starting configuration is characterized by a hydration environment showing significantly increased (as compared to the neutral DNA) reorientation of the molecular dipoles of water molecules located up to 7-8 $\AA$ from the adenine base on which a large fraction (57\%) of the ionization hole resides (see Methods). In the preferred orientation of the water molecules, the $\mathrm{H}_{2} \mathrm{O}$ dipole points away from the ionized base, with the lone-pair electrons of the oxygen atoms of the water pointing toward the positively charged base (see Methods), resulting in added attraction that lowers the total energy. Reaction-path simulations starting from other equilibrated configurations of the DNA radical cation yield results similar to those reported below. 


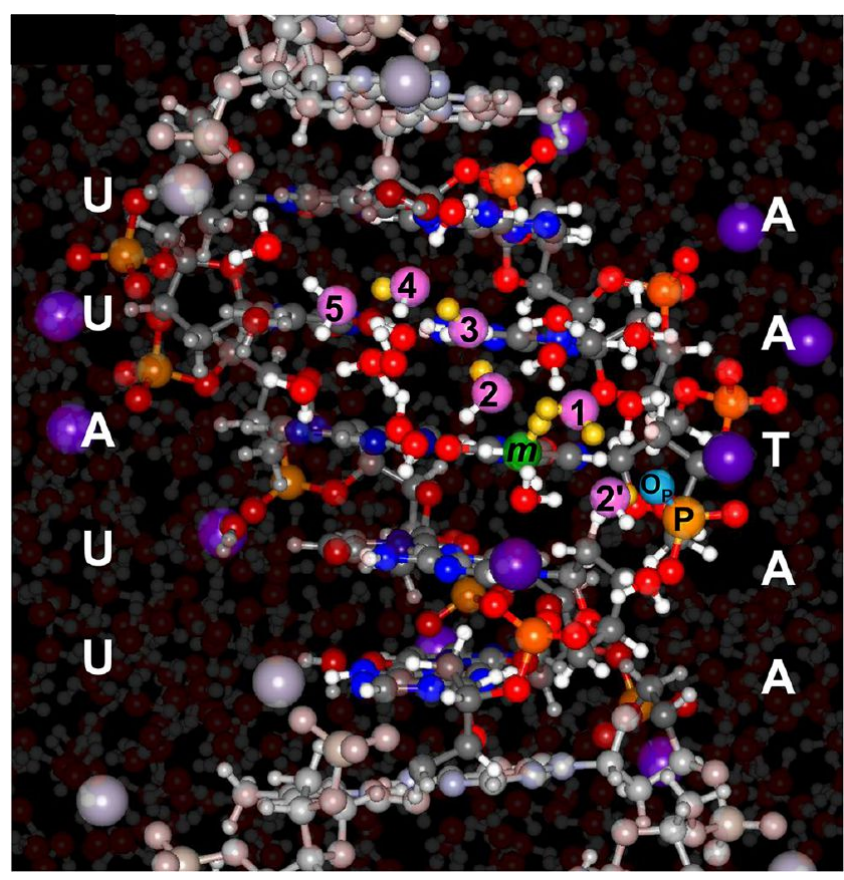

Figure 4. Atomic configuration of the DNA oligomer. Equilibrated and optimized atomic configuration of part of the 11-base-pair DNA duplex used as the starting configuration for the quantum simulation of the PCET deprotonation process. The central region (colored atoms), comprised of the five base-pair segment $\left(5^{\prime}\right.$-UUAUU- $\left.3^{\prime}\right) /\left(5^{\prime}\right.$-AATAA$\left.3^{\prime}\right)$, together with the corresponding sugar-phosphate backbone, sodium counterions, and hydrating water molecules, is treated quantum mechanically. The other six base-pairs (three on each end of the central region), together with the associated counterions and the rest of the water environment, are treated classically; some of the bases in the end regions are shown shaded in gray. The deprotonation reaction site is shown with the carbon atom of the methyl group (marked as " $\mathrm{m}$ ") of the proton donor thymine (T) colored in green. A backbone phosphate group, located between the donor $\mathrm{T}$ and the $5^{\prime}-\mathrm{A}$, is shown with an enlarged $\mathrm{P}$ atom colored orange, and one of its oxygen atoms, marked as $\mathrm{O}_{\mathrm{P}}$, is colored in light blue. Oxygen atoms of water molecules participating in the PCET process are colored pink, and transferred protons are colored yellow. Water molecules marked $1-5$ form a fragmented water-wire transporting the proton from the deprotonated methyl group, and the water molecule marked 2 ' and OP (along with water molecules 1 and 2) are part of an alternative channel where the proton is transported to the phosphate group $(\mathrm{P})$. In the configuration shown here, all sodium counterions (shown in deep purple) are located in the vicinity of the phosphate groups. In the quantum region, oxygen atoms are shown in red, nitrogen in dark blue, hydrogen atoms are colored white, phosphorus atoms are depicted in orange, and carbon atoms are colored dark gray. Stereographic images of are shown in section S2 of the Supporting Information.

Close-up views of the initial configuration used in investigation of the thymine deprotonation reaction and a sequence of reaction steps are shown in the bottom panels of Figure 5. The initial spatial distribution of the ionization hole (calculated as the spin density distribution) shown in the top panel of Figure 5A (superimposed on the central five-base-pair region) illustrates clearly that at the start of the deprotonation process the probability of finding the hole on $\mathrm{T}$ is vanishingly small. This is a result of the lower ionization potential of adenine than that of thymine: the calculated gas-phase difference between the adiabatic ionization potentials is $\Delta(\mathrm{aIP})$ $=0.96 \mathrm{eV}$. The hole distribution in the starting configuration is also evident in Figure 6 where the probability of finding the hole (nonzero spin) on the thymine $\left(P_{\sigma \mathrm{T}}\right)$ is displayed as a function of the reaction coordinate $D_{\mathrm{r}}=d_{\mathrm{C}-\mathrm{H}} / d_{\mathrm{H}-\mathrm{O}}$, with the interatomic distances defined in the upper left inset of Figure 6; initially, $D_{\mathrm{r}}^{0}=0.43\left(P_{\sigma \mathrm{T}}{ }^{0}=0.002\right)$. The proton-transfer process is simulated by use of the constrained first-principles $\mathrm{MD}$ (CFPMD) method (see Methods). In this method, all degrees of freedom are optimized for incremented values of a chosen constraint, taken here as the reaction coordinate, $D_{\mathrm{r}}$. The work required to displace the proton is determined as the integral of the force of constraint (calculated in the simulation) over the change in $D_{\mathrm{r}}$ taken along the reaction path from the initial configuration $D_{\mathrm{r}}^{0}$ (see the curve marked $\mathrm{E}$ in Figure 6). The value of the reaction coordinate at the transition state (TS) is denoted as $D_{\mathrm{r}}{ }^{*}$ with the proton-transfer work, or energy invested to reach this point, denoted as the TS barrier, $E^{*}$ (denoted also in the following as $\Delta_{\mathrm{a}}$ ). $D_{\mathrm{r}}{ }^{*}$ is defined such that for $D_{\mathrm{r}} \geq D_{\mathrm{r}}^{*}$ the system evolves without constraint to the product basin (i.e., from that point on the proton transfers freely to the neighboring water molecule). This procedure yields an upper-bound estimate of the reaction barrier because it does not include the entropy contribution $\left(-T \Delta S^{*}\right)$; that is, only the enthalpy part of the free-energy barrier is calculated. The inclusion of the entropy term (see the Supporting Information S4) and tunneling effects ${ }^{18,19}$ (see the Conclusion) will result in lowering of the effective proton-transfer barrier.

The process of proton transfer from the methyl group (proton donor, marked " $\mathrm{m}$ " in Figure $5 \mathrm{~A}$ ) to a neighboring proton accepting water molecule (marked " 1 " in Figure $5 \mathrm{~A}$ ) entails motion of the acceptor $\mathrm{H}_{2} \mathrm{O}$ toward the donor (see Figure $5 \mathrm{~B}$ and $\mathrm{C}$ ) expressed as a compression of $d_{\mathrm{C}-\mathrm{O}}$ (Figure 5 and Figure 6). The close-coupling between the proton and electron transfer from $\mathrm{T}$ to $\mathrm{A}$ (or equivalently hole transfer from $A$ to $T$ ) processes is evident from inspection of Figure $5 \mathrm{~A}-\mathrm{D}$ and Figure 6 . The increasing probability of finding the hole (i.e., excess spin, $\sigma \neq 0$ ) on the thymine is strongly correlated with the approach to the TS barrier, which occurs at a constraint value $D_{\mathrm{r}}{ }^{*}=1.4$ where the hole fraction achieves a value of $P_{\sigma \mathrm{T}}\left(D_{\mathrm{r}}^{*}\right)=0.65$ (see Figure 6), demonstrating unambiguously PCET characteristics. The TS barrier is found to be $\Delta_{\mathrm{a}} \approx 0.85 \mathrm{eV}$, and the approach to, and passage over, the barrier is accompanied by a rebound of the acceptor water molecule (see the upward turn of $d_{\mathrm{C}-\mathrm{O}}$ in Figure 6 as the TS barrier is approached). This rebound of the acceptor molecule is coupled with the decrease in the distance between this water molecule and a neighboring one (marked " 2 " in Figure 5A), culminating in the spontaneous formation (i.e., with no applied constraint force) of a Zundel ion, $\mathrm{H}_{5} \mathrm{O}_{2}^{+}$(see Figure 5D and the first local minimum marked $\mathrm{H}_{5} \mathrm{O}_{2}{ }^{+}$in Figure 6); formation of the Zundel ion is accompanied by a lowering of the energy by $0.42 \mathrm{eV}$ (with reference to the TS barrier). This process is accompanied by a further increase in the T-hole probability $P_{\sigma \mathrm{T}}$ $\left(D_{\mathrm{r}}=1.9\right)=0.86$; the spin distribution on the thymine at this state of the process is shown in the inset at the lower right side of Figure 6. During the deprotonation process, the WatsonCrick hydrogen bonds between the reacting thymine and its paired adenine remain essentially unchanged (see figure in S6 of the Supporting Information).

The PCET process described here is concerted and occurs on the ground-state adiabatic Born-Oppenheimer potential energy surface; the excited states of the simulated DNA oligomer radical cation are separated by a sufficiently large gap, particularly as the hole fraction on the thymine increases. Furthermore, the PCET process is orthogonal (bidirectional) ${ }^{6}$ 


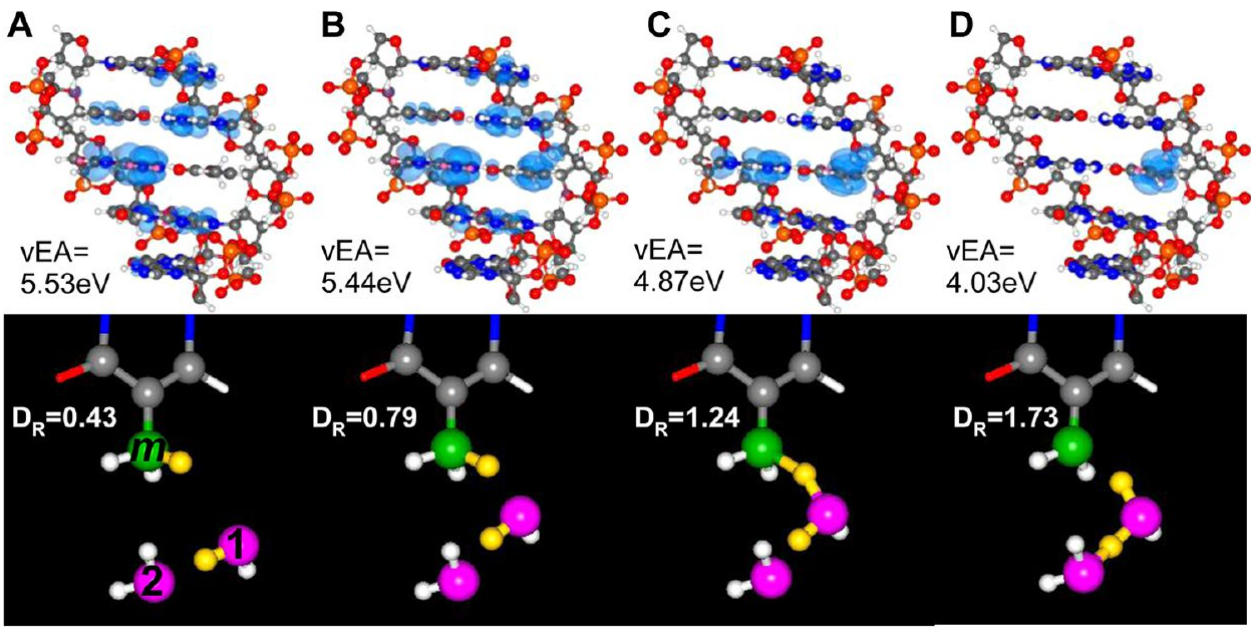

Figure 5. Evolution of the PCET deprotonation process. Bottom panel: A close-up view of the PCET reaction region, tilted with respect to the orientation given in Figure 4 to gain visual perspective. (A) The oxygen atoms of the two water molecules that mediate the proton transfer from the methyl $\left(\mathrm{CH}_{3}\right)$ donor (C atom depicted as the green sphere marked " $\mathrm{m}$ ") are denoted in pink (with numbers " 1 " and " 2 "), and the two protons participating in this stage of the process are colored yellow. This figure corresponds to the start of the PCET process with the reaction coordinate $D_{\mathrm{r}}$ $=0.43$. Top panel: Probability spin distribution, or equivalently hole distributions (blue colored isosurface drawn at a 0.8 contour level), superimposed on the atomic configuration of the central 5-base-pair region, showing that immediately after ionization the probability of finding the hole on $\mathrm{T}$ is negligible, with the largest fraction of the hole ( $\sim 57 \%)$ localized on the A base complementary to $\mathrm{T}$ (compare Figure 4). In (B)-(D), evolution of the PCET process for progressively larger values of the reaction coordinate: $D_{\mathrm{r}}=0.79$ (in B), 1.24 (in C), and 1.73 in (D). Initially (B) the $\mathrm{H}_{2} \mathrm{O}(1)$ molecule moves toward the nearest $\mathrm{H}$ atom of the methyl group. An hydronium $\left(\mathrm{H}_{3} \mathrm{O}^{+}\right)$ion anchored to the methyl group is formed as the TS barrier is approached (C), leading subsequently to the unactivated formation of a Zundel ion in (D). The electron transfer process from $\mathrm{T}$ to A (equivalently, transfer of the hole from A to T) that accompanies the nuclear configurational changes is documented in the sequence (A)-(D) in the top panels. It exhibits an essentially complete localization of the hole on $\mathrm{T}$ as the proton is transferred to the neighboring water molecules, thus illustrating the concerted nature of the PCET process. The values in the bottom panels of (A)-(D) are for the vertical electron affinities (vEA), calculated for the corresponding configurations as the difference between the total energies of the ionized and neutral DNA. The clear decreasing trend of the $\mathrm{vEA}$, correlating with the higher ionization potential of $\mathrm{T}$ than $\mathrm{A}$, could allow monitoring of the PCET process.

with the transferred proton and electron moving to different locations. That is, a neighboring water molecule is the initial proton acceptor, and the neighboring adenines are the electron acceptor (or equivalently stated, the thymine is the hole acceptor). Comparison between the PCET described above and the one occurring in a DNA configuration prepared such that the hole (radical cation) is initially strongly localized on the adenine complementary to the deprotonating $\mathrm{T}$ (to a larger degree than in the case shown in Figures 5 and 6; that is, 93\% as compared to $57 \%$, respectively) revealed essential similarity between the two (TS barriers of $\Delta_{\mathrm{a}} \approx 0.85$ and $0.87 \mathrm{eV}$, respectively, compare Figure 6 and Figure 10 in the Methods section), leading to the conclusion that the PCET deprotonation reaction is insensitive to the initial degree of ionizationhole spatial localization (see Methods). In both cases, the deprotonation processes that proceed by movements of water molecules that maintain as much as possible the integrity of the hydrogen-bonding network (that is, with no large elongations of hydrogen bonds) result in lower activation energy barriers.

The simulations revealed several PCET channels, some with a dependence on the specific counterion configuration in the vicinity of the relevant nucleobases; in this Article, we consider only configurations where the counterions are located in the vicinity of the backbone phosphate groups. In all cases, irreversibility of the PCET reaction is achieved upon eventual assimilation of the proton into the surrounding hydrating environment.

A PCET channel illustrating assimilation of the proton in the hydrating environment is shown in Figure 7. This process starts from the configuration shown in Figure 7A (the same as the one displayed in Figure 4, but only with the participating water molecules denoted by numbers). Close-in views of the protontransfer region are shown in Figure $7 \mathrm{~B}-\mathrm{F}$. As discussed above (see Figures 5 and 6), in the first step a proton on the methyl group of the donor thymine (see Figure $7 \mathrm{~B}$ ) transfers to a neighboring water molecule leading to formation of a Zundel ion $^{25-31}$ comprised of the transferred proton and the water molecules marked " 1 " and " 2 " (see Figure 7C), with the hole fraction on $\mathrm{T}$ achieving a value $P_{\sigma \mathrm{T}}=0.86$. Subsequently, the proton transports to the hydrating water environment in a process that involves a segmented "water wire"28,30,31 comprised here of alternating Zundel ions and a loosely bound $\left(\mathrm{H}_{7} \mathrm{O}_{3}{ }^{+}\right)$ion (see Figure $7 \mathrm{E}$ ). The transport processes starting from the Zundel configuration (Figure 7C) and ending with the one involving water molecules " 4 " and " 5 " (see Figure $7 F$ ) encounter very small energy barriers. The formation of the second Zundel ion (see Figure 7D) involves a barrier of merely $0.04 \mathrm{eV}$ with a subsequent energy lowering by $0.17 \mathrm{eV}$ (see Figure 6), and formation of the third Zundel ion (Figure 7F) entails a barrier of $0.07 \mathrm{eV}$. Accompanying the formation of the second Zundel ion (Figure 7D), the hole fraction on $\mathrm{T}$ increases to $P_{\sigma \mathrm{T}}=0.91 .^{32}$ Proton transfer culminating in solvation of the donated proton in the surrounding water underlies the irreversibility of the PCET reaction because the hydration environment represents a vast "entropic reservoir" inhibiting reversal of the reaction.

The proton transport mechanism that we find can be referred to as "structural diffusion", 33,34 consisting of transport of a structural defect and its hydrating coordination environment. This transport takes place through a sequence of proton transfers along a segmented "water wire", with the transport steps involving local small charged complexes, $\mathrm{H}_{3} \mathrm{O}^{+}, \mathrm{H}_{5} \mathrm{O}_{2}^{+}$, 


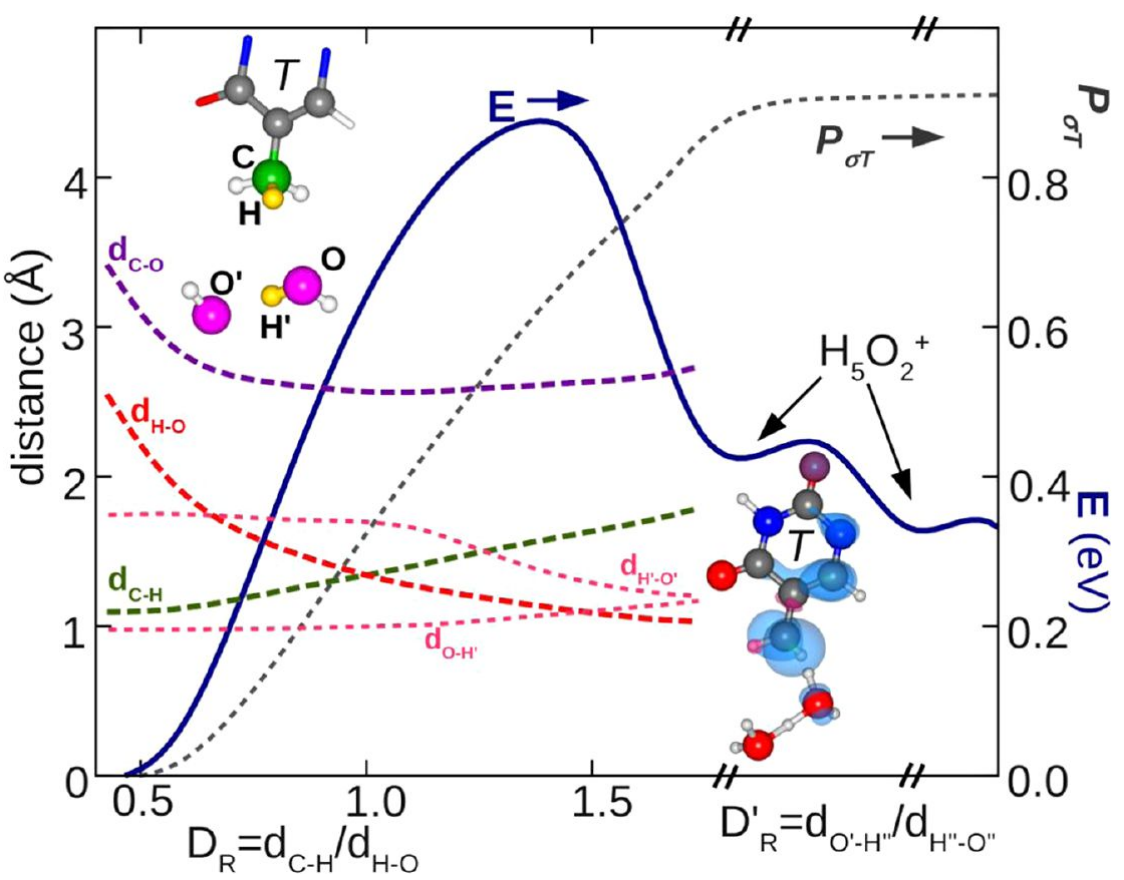

Figure 6. Variation of the hole fraction, energy, and distances during the PCET process. Changes of the energy associated with the proton transfer ( $E$, blue curve) and the probability for finding nonzero spin, $\sigma$, on $\mathrm{T}\left(P_{\sigma \mathrm{T}}\right.$, dotted black curve), equivalent to the fraction of the hole probability distribution on the deprotonating thymine, plotted as a function of the reaction coordinates $D_{\mathrm{r}}=d_{\mathrm{C}-\mathrm{H}} / d_{\mathrm{H}-\mathrm{O}}$ and $D_{\mathrm{r}}{ }^{\prime}=d_{\mathrm{O}^{\prime}-\mathrm{H}^{\prime}} / d_{\mathrm{H}^{\prime \prime}-\mathrm{O}^{\prime \prime}}$; $\mathrm{H}^{\prime \prime}$ is the left-

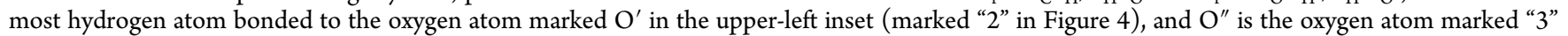
in Figure 4 belonging to the water molecule forming the second Zundel ion. Comparison of the variation of the energy and the correlated increase in $P_{\sigma \mathrm{T}}$ illustrates quantitatively the close-coupling between the proton and hole (or, equivalently, electron) transfer processes. Also shown (dashed curves) are the variations of certain interatomic distances as a function of $\mathrm{D}_{\mathrm{r}}$; for definition of these distances, see inset at upper left. At the start, $D_{\mathrm{r}}$ $\leq 0.7$, the variation of the reaction coordinate (and the energy, E) involves mainly the approach of the nearest $\mathrm{H}_{2} \mathrm{O}(1)$ molecule to the $\mathrm{CH}_{3}$ group (with little stretching of the $\mathrm{CH}$ bond). Also, the motion of $\mathrm{H}_{2} \mathrm{O}(1)$ does not involve stretching of the hydrogen bond to the neighboring $\mathrm{H}_{2} \mathrm{O}(2)$ molecule; see the flat behavior of $d_{\mathrm{H}^{\prime}-\mathrm{O}^{\prime}}$ and $d_{\mathrm{O}-\mathrm{H}^{\prime}}$ in fact, the decrease in $\mathrm{H}^{\prime}-\mathrm{O}^{\prime}$ occurs only for $D_{\mathrm{r}}>1.2$ corresponding to anchoring of $\mathrm{H}_{2} \mathrm{O}(1)$ to the $\mathrm{CH}_{3}$ group (see Figure $5 \mathrm{C}$ ). Movements of water molecules that maintain as much as possible the integrity of the hydrogen-bonding network lead to lower reaction activation energy barriers. The inset at the bottom right shows the spin (hole) distribution on the thymine radical (proton donor) at the end of the PCET process, exhibiting an almost complete hole localization on $\mathrm{T}\left(P_{\sigma \mathrm{T}}>0.9\right)$. The values of the hole fraction on T, corresponding to the PCET stages shown in Figure 4, are given in the Supporting Information.

and $\mathrm{H}_{7} \mathrm{O}_{2}{ }^{+}$. Significantly, the identity of the proton being transferred is most often not the same, and the transport process is incoherent, unlike the concerted proton transfers through extended structures described originally by von Grotthuss over two centuries ago. ${ }^{35}$ In this work, all nuclear degrees of freedom are treated classically. While quantization of the lightest nuclei (i.e., protons) is expected to enhance rate constants, it should not change the structural properties of the fragmented water wire and the essential nature of the protontransfer mechanism and pathways described here. This is, indeed, the lesson learned from studies of proton solvation and molecular mechanisms of excess proton transport in water. ${ }^{28-31}$ Finally, the cooperative nature of the water molecules hydrating $\mathrm{DNA}^{36}$ (generally exhibiting characteristics of confined fluids, i.e., preferential orientations, longer relaxation times, and "viscous dynamics") may influence the proton transport process and, consequently, deserves further study.

Figure 8 shows an alternative structural diffusion channel that leads to formation of a $\left[\mathrm{PO}_{4}{ }^{-} \mathrm{H}_{3} \mathrm{O}^{+}\right]$complex (see Figure $8 \mathrm{E}$ ). As before, starting with the initial configuration (Figures $8 \mathrm{~A}$, see also Figure 4), the process commences with an activated deprotonation of the methyl group (green sphere marked " $m$ " in Figures 8A and B) of the thymine and formation of a Zundel ion (comprised of the water molecules marked 1 and 2, Figure $8 \mathrm{C}$; see also Figures 5 and 6). At this stage, instead of following the water-wire path described in Figure 7, a proton is transferred (from the Zundel ion) to form another Zundel ion comprised of water molecules " 1 " and " 2 " (see Figure 8D); this process involves an energy barrier of only $0.1 \mathrm{eV}$, and a subsequent energy lowering of $0.2 \mathrm{eV}$. Fragmentation of this Zundel ion entails a TS energy barrier of $0.3 \mathrm{eV}$, and subsequent anchoring of the $\mathrm{H}_{3} \mathrm{O}^{+}$ion to the phosphate group (Figure $8 \mathrm{E}$ ) results in a decrease in energy by $0.28 \mathrm{eV}$. As before, eventually, the excess proton originating with the methyl-deprotonation reaction is transferred to the water reservoir.

\section{CONCLUSION}

The experiments and theoretical simulations demonstrate conclusively that the critical first step in the oxidative mutation of thymine is proton-coupled electron transfer. The experimentally observed isotope effect unambiguously demonstrates that cleavage of the methyl group carbon-hydrogen bond occurs in the rate-determining step leading to $T$ mutation. The first-principles quantum mechanical simulations show that localization of the hole on $\mathrm{T}$ occurs concurrently with the loss of the proton. The hole density on $\mathrm{T}$ increases from an initial vanishing value to about $70 \%$ at the reaction transition state (with an activation barrier $\Delta_{\mathrm{a}} \approx 0.85 \mathrm{eV}$ ), unambiguously revealing a bidirectional concerted PCET process that avoids high-energy intermediates. ${ }^{20}$ The marked decrease in the DNA vertical electron affinity (vEA, see bottom panels in Figure 5A- 


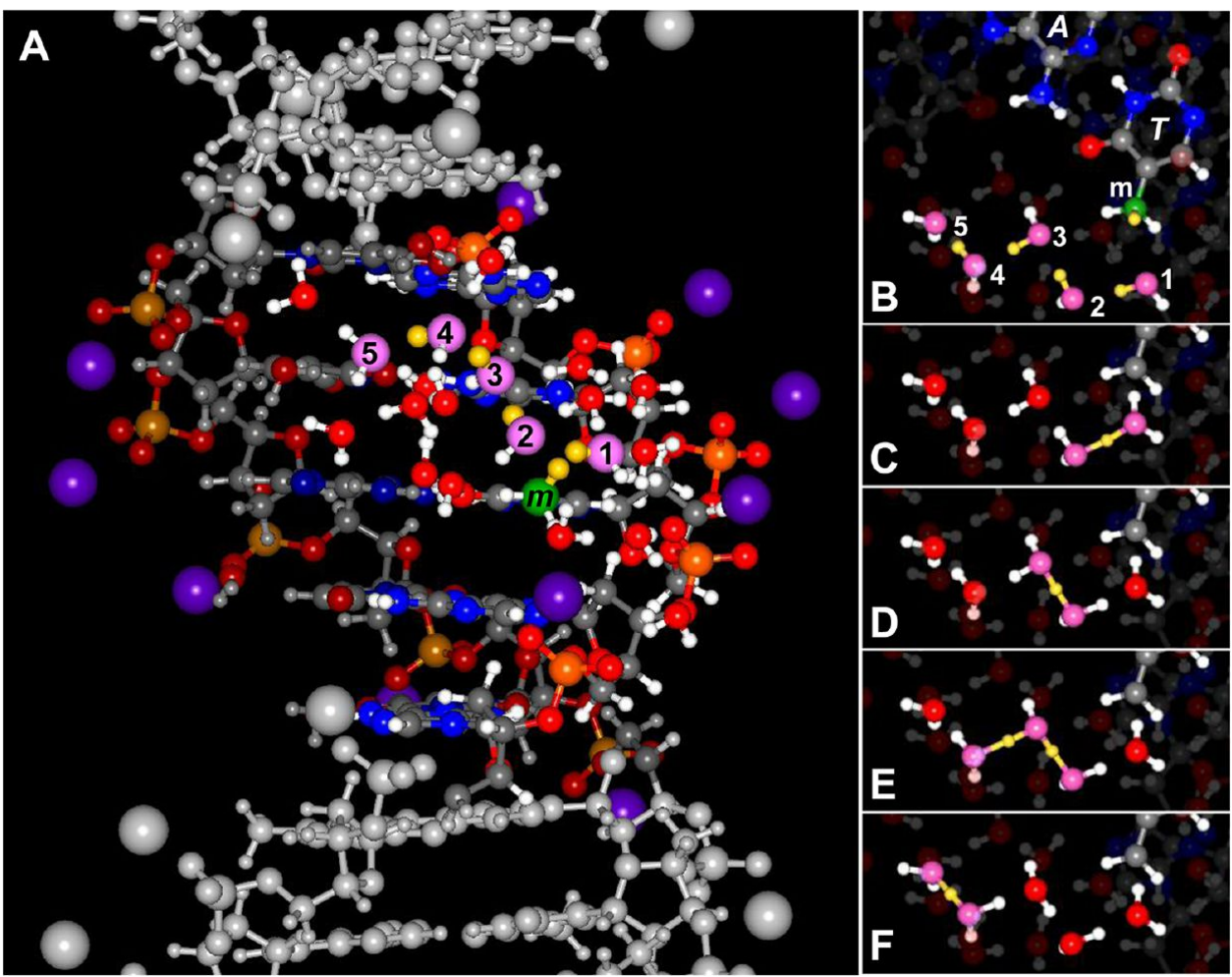

Figure 7. Steps in the thymine deprotonation mediated by a segmented water wire. (A) Atomic configuration (side-view) of part of the 11-base-pair DNA duplex used in the quantum simulations of the PCET deprotonation process; see caption of Figure 4. The oxygen atoms of five water molecules participating directly in the transfer of a proton (originating on the methyl group of $\mathrm{T}$, colored green) to the hydration environment are colored pink and are marked $1,2, \ldots 5$. The segmented water-wire proton transfer is described by the configurations shown in (B) $-(\mathrm{F})$, with the one in (B) extracted from the initial configuration shown in (A); the viewpoint in $(B)-(F)$ is tilted with respect to the one shown in (A). The activated initial deprotonation step is the same as that described in Figure $5 \mathrm{~B}-\mathrm{D}$, leading to the spontaneous formation of a Zundel ion (C, involving water molecules 1 and 2). The subsequent proton-transfer processes entail very small (thermal) energy barriers. Dissociation of the Zundel ion (C) leads to formation of a successive Zundel ion (involving molecules 2 and 3, shown in D), followed by formation of a 3-water-molecule wire segment $\left(\mathrm{H}_{7} \mathrm{O}_{3}{ }^{+}\right.$, shown in $\mathrm{F}$, involving water molecules 3, 4, and 5). The process culminates with dissociation of the wire segment resulting in formation of a Zundel ion as shown in $\mathrm{F}$ comprised of water molecules 5 and 6. Variation in the energy (up to formation and dissociation of the second Zundel ion (D)) is given in Figure 6. Interatomic distances in panels B-F can be found in Supporting Information S5.

D) predicted to occur during the proton-transfer processes correlates strongly with the sharp increase of the hole fraction on thymine (see $P_{\sigma \mathrm{T}}$ in Figure 6), thus suggesting possible timedependent electron spectroscopy exploration of the temporal evolution of the PCET mechanism in this system.

The parallel experimental and theoretical results presented herein advance our understanding of the microscopic mechanism underlying the oxidative reactions occurring at thymine in duplexes containing only A/T base pairs. However, theoretical calculations of the rate of the predicted PCET deprotonation reaction and of the measured kinetic isotope effect (KIE) are outside the scope of this Article and remain an outstanding challenge. Such calculations may adopt different methodologies: (i) simple tunneling corrections ${ }^{19-21,37}$ to "passage over the barrier" estimates, which result in higher values for the PCET reaction rate and the KIE factor than those obtained from the difference between the zero-point vibrational frequencies of $\mathrm{H}$ and $\mathrm{D}$ atoms (which yield a KIE of 6.2 for $\mathrm{C}-$ $\mathrm{H}$ bond cleavage reactions ${ }^{37}$ ); (ii) calculations within the equilibrium solvation framework that use modern multidimensional tunneling techniques to calculate the tunneling correction; $^{38,39}$ and (iii) calculations that treat the protontransfer $(\mathrm{PT})$ reaction as driven by configurational changes in the surrounding (hydrogen-bonded) environment. This is a feature of much modern work on processes involving PT reactions ${ }^{40}$ in which the reaction activation free energy is largely determined by environmental (solvent) reorganization. These treatments apply to proton-transfer reactions in the tunneling regime. Key ingredients in this picture include a complete quantum character for the proton motion (even when tunneling does not occur) and the identification of a solvent coordinate as the reaction coordinate. Modern treatments of PCET reactions and their application to the evaluation of KIE factors include: electronic coupling between the electron transfer donor and acceptor; coupling with the solvent; and vibrational overlap for the transferring proton between the vibrational levels in the initial state and in the final state. ${ }^{41}$

Dire consequences result when DNA replication goes awry. Aerobic organisms are continuously subjected to exogenous and endogenous oxidative stress causing one-electron oxidation of DNA and the generation of mutated nucleobases. In normal DNA, mutation occurs primarily at guanine, but in A/T-rich DNA mutations occur at thymine. Genomic instability during the early stages of cancer development has recently been associated with replication fork stalling at A/T-rich fragile regions, specifically at site FRA16C that contains segments comprised of 99 contiguous $\mathrm{A} / \mathrm{T}$ and $\mathrm{T} / \mathrm{A}$ base pairs. Oxidative damage at these sites is expected to yield thymine mutations known to cause replication stalling. Our investigation of the photoinduced oxidation of model oligomers provides a first 

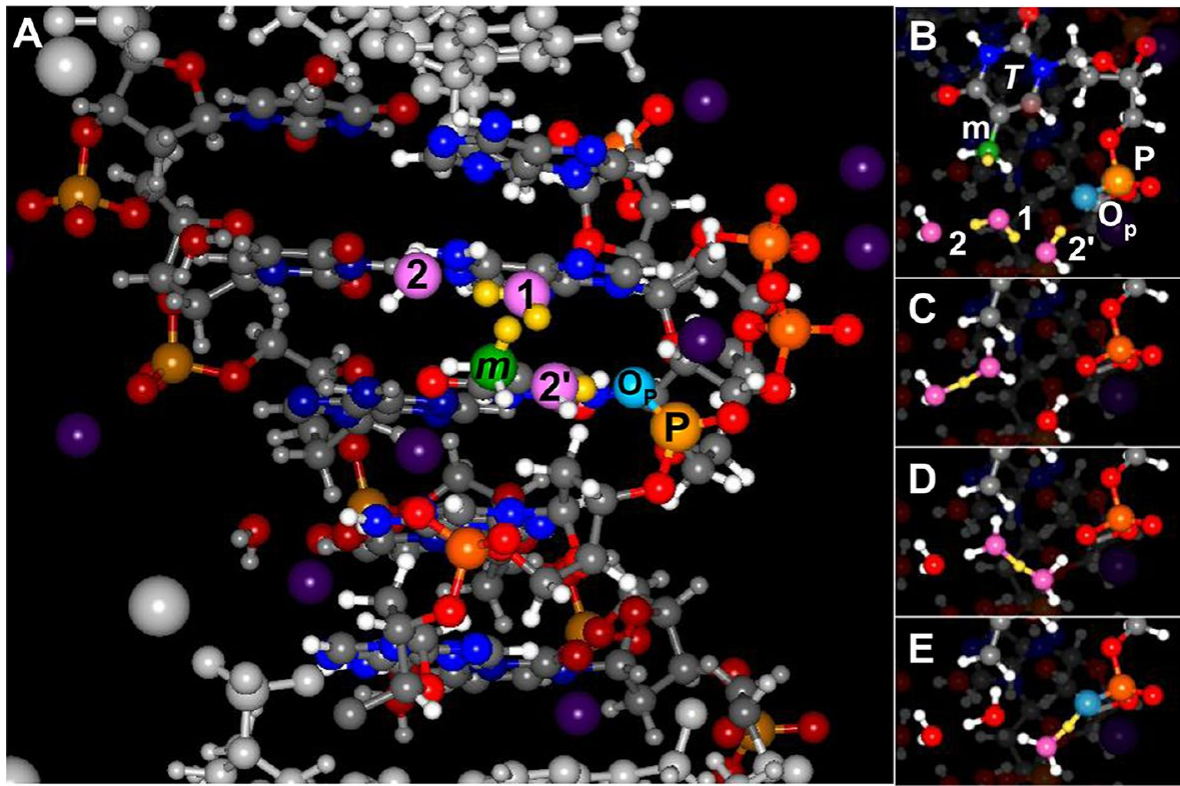

Figure 8. Steps in the thymine deprotonation mediated by water and anchoring at a phosphate group. (A) Atomic configuration (side-view) of part of the 11-base-pair DNA duplex used in the quantum simulations of the PCET deprotonation process; see caption of Figure 4. The oxygen atoms of three water molecules (oxygen atoms colored pink and marked 1,2, and $\left.2^{\prime}\right)$ and an oxygen of the phosphate group $\left(\mathrm{O}_{\mathrm{P}}\right.$, colored light blue) directly participating in the transfer of a proton (originating on the methyl group of $\mathrm{T}$, colored green) to the phosphate group (P) are highlighted. The proton transfer is described by the configurations shown in (B) - (E), with the one in (B) extracted from the initial configuration shown in (A); the viewpoint in $(\mathrm{B})-(\mathrm{F})$ is tilted with respect to the one shown in $(\mathrm{A})$. The activated initial deprotonation step is the same as described in Figure $5 \mathrm{~B}-$ $\mathrm{D}$ (see also Figure 7B), leading to the spontaneous formation of a Zundel ion (C, involving water molecules 1 and 2). Subsequently a proton is transferred (from the Zundel ion) to form another Zundel ion comprised of water molecules 1 and 2' (D). Activated dissociation of the Zundel leads to anchoring of the $\mathrm{H}_{3} \mathrm{O}^{+}$ion to the phosphate group (E).

look at the detailed molecular mechanism for this thymine mutation process.

\section{METHODS}

Experiment. The DNA oligomers shown in Figure 2 were prepared, purified, and analyzed by standard methods. ${ }^{42-44}$ The oligomers were irradiated at $350 \mathrm{~nm}$ (where the Aq absorbs) for $2 \mathrm{~min}$, and then the irradiated samples were treated with piperidine or endo III enzyme, which cause strand cleavage at the sites of mutated bases. ${ }^{23}$

Theory. 1. Computational Methods. The FPMD method that we use for the central five base-pair quantum region (Figure 2) including the sugar-phosphate backbone, associated counterions, and hydrating water molecules has been specifically formulated for treating charged systems. ${ }^{45}$ The method calculates the electronic structure by employing the Kohn-Sham density-functional theory (DFT) with the use of a plane wave-basis (62 Ry kinetic energy cutoff), in conjunction with soft pseudopotentials ${ }^{46}$ and the Perdew-BurkeErnzerhof (PBE) functional ${ }^{47}$ in the generalized gradient approximation (GGA) to the exchange-correlation energy. The other six base-pairs (three on each end of the central region, see Figure 4) of the simulated 11-base-pair DNA oligomer as well as their associated counterions and water molecules in the rest of the hydration environment are treated classically (see the Supporting Information), and they are coupled to the central QM region through the use of a hybrid QM/MM approach ${ }^{4}$ (see Supporting Information S1A) where MM (molecular mechanics) corresponds to the classically treated part of the simulated system.

In the constrained first-principles molecular dynamics (CFPMD) simulations, one judiciously selects first a reaction coordinate, which can be a distance between two chosen atoms, the ratio between certain interatomic distances, an angle subtended by atoms pertinent to the reaction mechanism, or a combination of interatomic distances and/or angles. Subsequently, the chosen reaction coordinate is assigned a selected value, and all of the degrees of freedom of the system are allowed to relax freely (to a local energy minimum) but subject to the

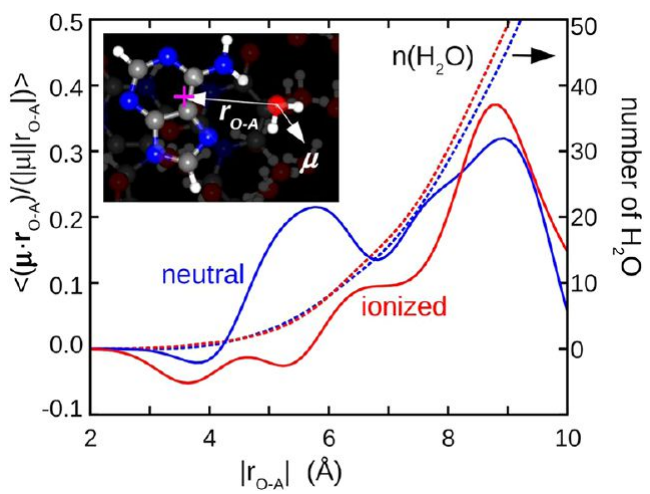

Figure 9. Preferential orientation of hydrating water molecules. Variation of the dipole orientational order parameter $\mathbf{O}\left(\boldsymbol{\mu}, r_{\mathrm{O}-\mathrm{A}}\right)$. The blue curve shows the behavior for neutral (un-ionized) DNA, and the one in red is for a singly ionized DNA (with $57 \%$ of the hole localized on the adenine that is complementary to the deprotonating $\mathrm{T}$ ). The corresponding dashed lines give the number of water molecules contributing to the average at a distance $r_{\mathrm{O}-\mathrm{A}}$; see $n\left(\mathrm{H}_{2} \mathrm{O}\right)$ on the right axis. While the number of water molecules in the vicinity of the DNA molecule remains the same (before and after ionization), the orientations of the water molecules with respect to the charged region (measured by the direction of the molecular diploes) are found to undergo a significant change. The definitions of the position vector $r_{\mathrm{O}-\mathrm{A}}$ the dipole moment of a water molecule, $\mu$, and the position of the center of excess charge (CEC, denoted by a " + ") are given in the inset.

chosen constraint (that is, the prescribed value of the reaction coordinate), and the "force-of-constraint", $F_{\text {const }}$ is determined. This process is repeated for successive incremented variations of the reaction coordinate until the system overcomes the TS reaction barrier and reaches the product basin. The integral of $F_{\text {const }}$ over the values of 


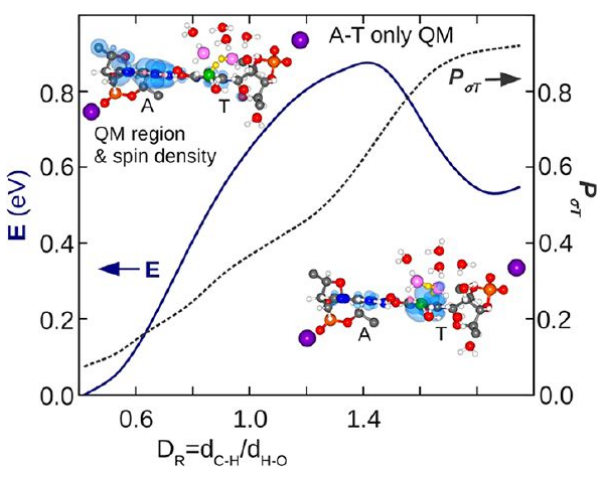

Figure 10. Deprotonation of thymine in ionized DNA with a strongly localized hole on A: Variation of the hole fraction and energy during the PCET process. Same as Figure 6 but for the case of a single base pair in the quantum mechanical region, $\left\{\mathrm{d}\left(5^{\prime}\right.\right.$-TTA[UUAUU]ATT $\}$ $\left.\left.3^{\prime}\right)\right\} /\left\{\right.$ d $\left(3^{\prime}\right.$-AAT[AATAA]TAA $\left.\left.\}-5^{\prime}\right)\right\}$. Changes of the energy associated with the proton transfer (E, blue curve) and the probability for finding nonzero spin, $\sigma$, on $\mathrm{T}$ ( $P_{\sigma \mathrm{T}}$, dotted black curve), equivalent to the fraction of the hole probability distribution on the deprotonating thymine, plotted as a function of the reaction coordinates $D_{\mathrm{r}}=d_{\mathrm{C}-\mathrm{H}} /$ $d_{\mathrm{H}-\mathrm{O}}$. Comparison of the variation of the energy and the correlated increase in $P_{\sigma \mathrm{T}}$ illustrates quantitatively the close-coupling between the proton and hole (or, equivalently, electron) transfer processes, with the reaction characteristic resembling closely those shown in Figure.6. The insets in the upper left and lower right depict the central (quantum) region initially $\left(D_{\mathrm{r}}=0.42\right)$ and after going over the TS reaction barrier leading to formation of a Zundel ion $\left(D_{\mathrm{r}}=1.72\right)$, respectively. Superimposed on the atomic configurations are the probability spin distribution, or equivalently hole distributions (blue colored isosurface drawn at a 0.8 contour level). The color designations in the insets are as follows: $\mathrm{C}$ atoms, gray; $\mathrm{N}$, blue; $\mathrm{P}$, orange; $\mathrm{O}$, red; $\mathrm{H}$, white; $\mathrm{C}$ atom of deprotonating $\mathrm{CH}_{3}$ group on $\mathrm{T}$, green; oxygen atoms of $\mathrm{H}_{2} \mathrm{O}$ molecules participating in the PCET process, pink; protons participating in the transfer processes, yellow.

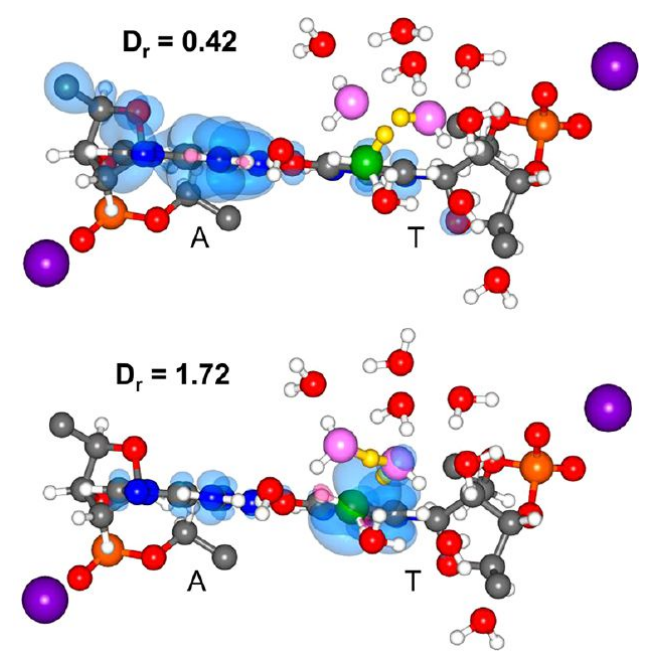

Figure 11. Enlargements of the insets shown in Figure 10. Superimposed on the initial $\left(D_{\mathrm{r}}=0.42\right.$, upper panel $)$ and the Zundel ion formation $\left(D_{\mathrm{r}}=1.72\right.$, lower panel $)$ atomic configurations, we show the corresponding spin distributions (blue colored isosurfaces drawn at the $80 \%$ spin density contour level) exhibiting a hole (radical cation) on $\mathrm{A}$ (upper panel) and a radical on $\mathrm{T}$ (bottom panel).

the reaction coordinate gives the variation of the reaction energetics (in particular, the reaction barrier height) during the reactive event, which, together with the corresponding evolution of the structural (geometrical) parameters of the system, describes the reaction mechanism.
2. Water Reorganization and Hole Distribution. In the following, we address topics that pertain to theoretical methodological issues concerning the physical chemistry of DNA. First, we explore the response of the DNA hydration layer to ionization of the molecule, that is, removal of an electron that leaves behind a positive hole (a radical cation). Charge solvation (in particular hydration) is a fundamental property of liquids, and a molecular level understanding of this process is imperative for elucidating energetics, stability, dynamics, and reaction pathways in liquids. This topic has been the subject of numerous studies in the context of hole creation, stabilization, and migration in DNA, and the effects of reorganization of the hydrating molecules and hydrating environment fluctuations on these processes. ${ }^{12,48-60}$ Subsequently, we address another topic pertinent to the nature of an ionization hole in DNA and its affect on chemical processes in ionized DNA. Specifically, we discuss the effect of the spatial localization of a hole in DNA on the deprotonation PCET mechanism. The energetics of an excess charge (hole or excess electron) in DNA is a complex problem because it is influenced by the hydrating environment as well as by the solvated counterions. ${ }^{12,48-60}$ Additionally, recent discussion of this topic attempts to address certain computational issues (particularly the DFT self-interaction) pertinent to the hole localization issue. ${ }^{61-63}$

i. Local Water Reorganization in lonized. To further characterize the starting ionized DNA configuration, we explored the response of the hydration environment to the ionization of the DNA molecule. As elaborated below, the ionization hole (excess positive charge brought about by the removal of an electron) is calculated to be distributed initially with the dominant fraction (57\%) localized on the adenine complementary to the $\mathrm{T}$ whose methyl group serves as the deprotonation site (see Figure 4, upper panels of Figure 5A, and section S3 in the Supporting Information). For the method of preparation of the system, see section S1 B. To investigate the change in the water environment upon ionization, we compare in Figure 9 the behavior of a dipole orientational order parameter, defined as

$$
\mathrm{O}\left(\left(\boldsymbol{\mu}, \boldsymbol{r}_{\mathbf{O}-\mathbf{A}}\right)=\left\langle\left(\boldsymbol{\mu} \cdot \boldsymbol{r}_{\mathbf{O}-\mathbf{A}}\right) /\left(\boldsymbol{\mu} \| \boldsymbol{r}_{\mathbf{O A}} \mid\right)\right\rangle=\left\langle\cos \beta\left(\boldsymbol{\mu}, \boldsymbol{r}_{\mathbf{O}-\mathbf{A}}\right)\right\rangle\right.
$$

for the neutral and ionized systems. In eq $1, \beta$ is the angle between the dipole moment, $\boldsymbol{\mu}$, of an $\mathrm{H}_{2} \mathrm{O}$ molecule whose oxygen atom $\left(\mathrm{O}\right.$, at $\left.\boldsymbol{r}_{\mathrm{O}}\right)$ is located at $r_{\mathrm{O}-\mathrm{A}}\left(=r_{\mathrm{CEC}}-r_{\mathrm{O}}\right)$ with respect to the center of the excess charge (CEC) in the ionized adenine base (taken as the base on which the largest fraction of the ionization hole (excess spin, $\sigma \neq 0$ ) resides); see inset in Figure 9. The vector position of the CEC is defined as $r_{\text {CEC }}$ $=\sum_{k} r_{k} \Delta \rho_{k} / \sum_{k} \Delta \rho_{k}$, which is the sum of the position vectors of the atoms in the base weighted by the fraction of the ionization hole on each atomic site $k$ (with $\Delta \rho_{k}$ determined by a Bader analysis ${ }^{64,65}$ of the charge density difference on the $\mathrm{A}$ base, between the neutral and ionized DNA). In eq $1,\langle\ldots\rangle$ indicates averaging over the water molecules located at a distance $r_{\mathrm{O}-\mathrm{A}}$ from the CEC of the A base. The solid lines in Figure 9 show, respectively, the behavior of the neutral (blue) and singly ionized (red) states of the solvated DNA molecule. The dashed lines give the number of water molecules, $n\left(\mathrm{H}_{2} \mathrm{O}\right)$ (see right axis), contributing to the average at a distance $r_{\mathrm{O}-\mathrm{A}}$.

Inspection of $n\left(\mathrm{H}_{2} \mathrm{O}\right)$ for the two DNA charge states reveals that the number of water molecules in the vicinity of the DNA molecule remains the same (before and after ionization). On the other hand, the distribution of orientations of the water molecules with respect to the charged region is found to differ significantly from the one calculated for the neutral system. In particular, up to $r_{\mathrm{O}-\mathrm{A}} \approx 8 \AA$, corresponding to a hydration environment of $n\left(\mathrm{H}_{2} \mathrm{O}\right) \approx 25-27$, the values of the dipole orientation parameter $\mathrm{O}\left(\mu, r_{\mathrm{O}-\mathrm{A}}\right)$ are notably smaller for the ionized DNA case. This reflects larger angles between the molecular dipoles and $r_{\mathrm{O}-\mathrm{A}}$, corresponding to preferential reorientation of the protons of the water molecules away from the (positively charged) ionized region. We also note that in the ionized case $\mathrm{O}\left(\mu, r_{\mathrm{O}-\mathrm{A}}\right)<0$ for water molecules located in a region with $r_{\mathrm{O}-\mathrm{A}} \leq 6 \AA$ (containing about $n\left(\mathrm{H}_{2} \mathrm{O}\right) \leq 10$ molecules), reflecting a particularly strong dipole orientational polarization in the near hydration shell, with both protons of the water molecules in that close region pointing away from the ionized adenine. This preferential orientation, which results in water molecules oriented with their "negative end" (i.e., oxygen lone- 
pair) closer to the positively charged (ionized) base, results in added attraction that lowers the energy of the system and bootstraps the localization of the hole.

ii. PCET Reaction Starting from a Strongly Localized Hole. Here, we explore the dependence of the characteristics of the deprotonation process on the degree of initial hole localization. In the main text of this Article, we explored the deprotonation process for an energyoptimized ionized configuration of the solvated 11-base-pair DNA oligomer $\left(5^{\prime}\right.$-TTA[UUAUU]ATT $\}$ - $\left.\left.3^{\prime}\right)\right\} /\left\{\mathrm{d}\left(3^{\prime}\right.\right.$-AAT[ AATAA $\left.] \mathrm{TAA}\right\}$ $\left.\left.5^{\prime}\right)\right\}$, with the five base-pairs underlined and in bold treated quantum mechanically, prepared as described in section S1 B2. In the optimized initial configuration, $57 \%$ of the hole (excess spin) was localized on the middle adenine (A) (in the center of the quantum region), complementary to $\mathrm{T}$ (the deprotonation site). The deprotonation reaction was found to follow a concerted, bidirectional PCET process, with a transition-state activation barrier of $0.85 \mathrm{eV}$, leading to formation of a Zundel ion (see Figures 5 and 6), with the hole fraction on T achieving a value of $P_{\sigma \mathrm{T}}\left(D_{\mathrm{r}}{ }^{*}\right)=0.65$ at the top of the barrier, and further increasing to 0.86 upon formation of the Zundel ion.

To investigate the possible effect of hole-localization on the PCET deprotonation process, we prepared a system consisting of only a single $\mathrm{A} / \mathrm{T}$ base-pair treated quantum mechanically, $\left\{\mathrm{d}\left(5^{\prime}\right.\right.$-TTAUU[a] UUATT $\left.\left.\}-3^{\prime}\right)\right\} /\left\{\mathrm{d}\left(3^{\prime}\right.\right.$-AATAA[T] AATAA $\left.\left.\}-5^{\prime}\right)\right\}$ along with the corresponding sugar-phosphate groups, two sodium counterions, and 12 water molecules. See Supporting Information section 1B for details about preparation of this system. Following this procedure, we find $93 \%$ localization of the hole on the central adenosine with $7 \%$ residing on the complementary $\mathrm{T}$.

The results of the CFPMD simulation of the deprotonation reaction starting from the strongly localized-hole DNA configuration are shown in Figures 10 and 11. The TS barrier $(0.87 \mathrm{eV})$ is reached for a reaction coordinate $D_{\mathrm{r}}^{*}=1.45$, where the hole fraction on $\mathrm{T}$ achieves a value of $P_{\sigma \mathrm{T}}\left(D_{\mathrm{r}}{ }^{*}\right)=0.62$. When the Zundel ion is formed, the hole (spin density) localizes on the thymine, that is, $P_{\sigma \mathrm{T}}=0.92$. The excess spin distributions at the start of the simulation $\left(D_{\mathrm{r}}=0.42\right.$, upper left $)$ and at the Zundel ion formation stage $\left(D_{\mathrm{r}}=1.74\right.$, lower right $)$ are shown as the inset in Figure 10, and are enlarged in Figure 11. The results for the reaction pathway shown here portray a concerted PCET very close to that shown in Figure 6, which is for the case where the deprotonation starts from a less strongly localized hole $\left(P_{\sigma \mathrm{T}}=0.57\right)$. We conclude that the nature and characteristics of the deprotonation process are essentially independent of the degree of initial hole localization.

\section{ASSOCIATED CONTENT}

\section{S Supporting Information}

Data used to generate Figure 3. Computational methodology and techniques. Stereo images of Figure 4 of the main text. Fraction of spin (hole) distribution on bases. Constrained firstprinciples molecular dynamics (CFPMD) simulations. Distance variations (Figure 7). A-T hydrogen-bond distances before and after deprotonation. This material is available free of charge via the Internet at http://pubs.acs.org.

\section{AUTHOR INFORMATION}

\section{Corresponding Author}

uzi.landman@physics.gatech.edu; schuster@gatech.edu

\section{Present Address}

"Photosciences \& Photonics, Chemical Sciences and Technology Division, CSIR-NIIST, Trivandrum, Kerala - 695 019, India

\section{Notes}

The authors declare no competing financial interest.

\section{ACKNOWLEDGMENTS}

The work of U.L. and R.N.B. has been supported by the U.S. Office of Basic Energy Sciences under Contract ER45234, and in part by a grant from the U.S. Air Force Office for Scientific Research (AFOSR). Computations have been performed at NERSC, the National Energy Research Scientific Computer Center, National Energy Research Scientific Computing Center, which is supported by the Office of Science of the U.S. Department of Energy under Contract No. DE-AC0205CH11231, on Department of Defense computers through the high performance computing modernization program, and at the Center for computational materials science at the Georgia Institute of Technology. We thank the Vassar Woolley Foundation for financial support.

\section{REFERENCES}

(1) Weinberg, D. R.; Gagliargi, C. J.; Hull, J. F.; Murphy, C. F.; Kent, C. A.; Westlake, B. C.; Paul, A.; Ess, D. H.; McCafferty, D. G.; Meyer, T. J. Chem. Rev. 2012, 112, 4016.

(2) Meyer, T. J.; Huynh, M. H.; Thorp, H. H. Angew. Chem. 2007, 46, 5284 .

(3) Reece, S. Y.; Nocera, D. G. Annu. Rev. Biochem. 2009, 78, 673.

(4) Barnett, R. N.; Bongiorno, A.; Cleveland, C. L.; Joy, A.; Landman, U.; Schuster, G. B. J. Am. Chem. Soc. 2006, 128, 10795.

(5) Kryston, T. B.; Georgiev, A. B.; Pissis, P.; Georgakilas, A. G. Mutat. Res. 2011, 711, 193.

(6) Kumar, A.; Sevilla, M. D. Chem. Rev. 2010, 110, 7002

(7) Ghosh, A.; Joy, A.; Schuster, G. B.; Douki, T.; Cadet, J. Org. Biomol. Chem. 2008, 6, 916.

(8) Ozeri-Galai, E.; Lebofsky, R.; Rahat, A.; Bester, A. C.; Bensimon, A.; Kerem, B. Mol. cell 2011, 43, 122.

(9) Ma, K.; Qiu, L.; Mrasek, K.; Zhang, J.; Liehr, T.; Quintana, L. G.; Li, Z. Int. J. Mol. Sci. 2012, 13, 11974.

(10) Aller, P.; Rould, M. A.; Hogg, M.; Wallace, S. S.; Doublie, S. Proc. Natl. Acad. Sci. U.S.A. 2007, 104, 814.

(11) Ide, H.; Kow, Y. W.; Wallace, S. S. Nucleic Acids Res. 1985, 13, 8035.

(12) Kanvah, S.; Joseph, J.; Schuster, G. B.; Barnett, R. N.; Cleveland, C. L.; Landman, U. Acc. Chem. Res. 2010, 43, 280.

(13) Genereux, J. C.; Barton, J. K. Chem. Rev. 2010, 110, 1642.

(14) Steenken, S.; Jovanovic, S. V. J. Am. Chem. Soc. 1997, 119, 617.

(15) Joseph, J.; Schuster, G. B. Chem. Commun. 2010, 46, 7872.

(16) Kuttappan-Nair, V.; Samson-Thibault, F.; Wagner, J. R. Chem. Res. Toxicol. 2010, 23, 48.

(17) Curtin, D. Y. Rec. Chem. Prog. 1954, 15, 111.

(18) Thornton, E. K.; Thornton, E. R. In Isotope Effects in Chemical Reactions; Collins, C. J., Bowman, N. S., Eds.; Van Nostrand Reinhold: New York, 1970; p 213.

(19) Melander, L.; Saunders, W. H., Jr. Reaction Rates of Isotopic Molecules; Wiley: New York, 1980.

(20) Huynh, M. H. V.; Meyer, T. J. Proc. Natl. Acad. Sci. U.S.A. 2004, $101,13138$.

(21) Schuster, G. B. Acc. Chem. Res. 2000, 33, 253.

(22) Deeble, D. J.; Schuchmann, M. N.; Steenken, S.; Vonsonntag, C. J. Phys. Chem. 1990, 94, 8186.

(23) Burrows, C. J.; Muller, J. G. Chem. Rev. 1998, 98, 1109.

(24) There is a reproducible small increase in reaction at adenines in the deuterated sample. This is a result of suppression of the reaction at the $\mathrm{T}_{\mathrm{D} 3}$ position.

(25) Schuster, P.; Zundel, G.; Sandorfy, C. The Hydrogen Bond Recent Developments in Theory and Experiments. II. Structure and Spectroscopy; North-Holland: Amsterdam, 1976.

(26) Marx, D.; Tuckerman, M. E.; Hutter, J.; Parrinello, M. Nature 1999, 397, 601 .

(27) Cheng, H. P.; Barnett, R. N.; Landman, U. Chem. Phys. Lett. 1995, 237, 161.

(28) Mei, H. S.; Tuckerman, M. E.; Sagnella, D. E.; Klein, M. L. J. Phys. Chem. B 1998, 102, 10446.

(29) Marx, D.; Tuckerman, M. E.; Parrinello, M. J. Phys: Condens. Matter 2000, 12, A153. 
(30) Geissler, P. L.; Dellago, C.; Chandler, D.; Hutter, J.; Parrinello, M. Science 2001, 291, 2121.

(31) Hassanali, A.; Prakash, M. K.; Eshet, H.; Parrinello, M. Proc. Natl. Acad. Sci. U.S.A. 2011, 108, 20410.

(32) The spin probability is evaluated from the calculated wave functions by summing over nonoverlapping spheres drawn about the atoms of the thymine base. Consequently, our values for $P_{\sigma} T$ are lower bounds of the hole fraction.

(33) Marx, D.; Chandra, A.; Tuckerman, M. E. Chem. Rev. 2010, 110, 2174.

(34) Marx, D. ChemPhysChem 2006, 7, 1848.

(35) de Grotthuss, C. J. T. Ann. Chim. 1806, 58, 54.

(36) Spink, C. H.; Chaires, J. B. Biochemistry 1999, 38, 496.

(37) Bell, R. P. The Tunnel Effect in Chemistry; Chapman Hall: New York, 1980.

(38) Cui, Q.; Karplus, M. J. Am. Chem. Soc. 2002, 124, 3093.

(39) Truhlar, D. G.; Gao, J. L.; Alhambra, C.; Garcia-Viloca, M.;

Corchado, J.; Sanchez, M. L.; Villa, J. Acc. Chem. Res 2002, 35, 341.

(40) Kiefer, P. M.; Hynes, J. T. J. Phys. Org. Chem. 2010, 23.

(41) Hammes-Schiffer, S.; Hatcher, E.; Ishikita, H.; Skone, J. H.; Soudackov, A. V. Coord. Chem. Rev. 2008, 252, 384.

(42) Armstrong, R. W.; Gupta, S.; Whelihan, F. Tetrahedron Lett. 1989, 30, 2057.

(43) Gasper, S. M.; Schuster, G. B. J. Am. Chem. Soc. 1997, 119, 12762.

(44) Ahmadian, M.; Bergstrom, D. E. Nucleosides Nucleotides 1998, $17,1183$.

(45) Barnett, R. N.; Landman, U. Phys. Rev. B: Condens. Matter 1993, 48, 2081.

(46) Troullier, N.; Martins, J. L. Phys. Rev. B: Condens. Matter 1991, 43, 1993.

(47) Perdew, J. P.; Burke, K.; Ernzerhof, M. Phys. Rev. Lett. 1996, 77, 3865.

(48) Barnett, R. N.; Cleveland, C. L.; Joy, A.; Landman, U.; Schuster, G. B. Science 2001, 294, 567.

(49) Basko, D. M.; Conwell, E. M. Phys. Rev. Lett. 2002, 88.

(50) Kurnikov, I. V.; Tong, G. S. M.; Madrid, M.; Beratan, D. N. J. Phys. Chem. B 2002, 106, 7.

(51) Barnett, R. N.; Cleveland, C. L.; Landman, U.; Boone, E.; Kanvah, S.; Schuster, G. B. J. Phys. Chem. A 2003, 107, 3525.

(52) Voityuk, A. A.; Siriwong, K.; Rosch, N. Angew. Chem., Int. Ed. 2004, 43, 624 .

(53) Berlin, Y. A.; Kurnikov, I. V.; Beratan, D.; Ratner, M. A.; Burin, A. L. Long-Range Charge Transfer in DNA II 2004, 237, 1.

(54) Barnett, R. N.; Bongiorno, A.; Cleveland, C. L.; Joy, A.; Landman, U.; Schuster, G. B. J. Am. Chem. Soc. 2006, 128, 10795.

(55) Kubar, T.; Elstner, M. J. Phys. Chem. B 2008, 112, 8788.

(56) Kubar, T.; Kleinekathofer, U.; Elstner, M. J. Phys. Chem. B 2009, 113, 13107.

(57) Kubar, T.; Elstner, M. J. Phys. Chem. B 2009, 113, 5653.

(58) Vladimirov, E.; Ivanova, A.; Rosch, N. J. Phys. Chem. B 2009, 113,4425 .

(59) Burin, A. L.; Uskov, D. B. J. Chem. Phys. 2008, 129, 025101.

(60) Kravec, S. M.; Kinz-Thompson, C. D.; Conwell, E. M. J. Phys. Chem. B 2011, 115, 6166.

(61) Mantz, Y. A.; Gervasio, F. L.; Laino, T.; Parrinello, M. J. Phys. Chem. A 2007, 111, 105.

(62) Mantz, Y. A.; Gervasio, F. L.; Laino, T.; Parrinello, M. Phys. Rev. Lett. 2007, 99, 058104.

(63) Kubar, T.; Elstner, M. J. Phys. Chem. B 2010, 114, 11221.

(64) Bader, R. F. W. Atoms in Molecules: A Quantum Theory; Oxford University Press: New York, 1990.

(65) Tang, W.; Sanville, E.; Henkelman, G. J. Phys.: Condens. Matter 2009, 21. 